\title{
A case-control study on risk factors of breast cancer in China
}

\author{
Ya-Li Xu', Qiang Sun ${ }^{1}$, Guang-Liang Shan², Jin Zhang ${ }^{3}$, Hai-Bo Liao ${ }^{4}$, Shi-Yong Lí, Jun Jiang ${ }^{6}$, \\ Zhi-Min Shao ${ }^{7}$, Hong-Chuan Jiang ${ }^{8}$, Nian-Chun Shen ${ }^{9}$ Yue Shi ${ }^{10}$, Cheng-Ze Yu ${ }^{11}$, Bao-Ning Zhang ${ }^{12}$, \\ Yan-Hua Chen ${ }^{13}$, Xue-Ning Duan ${ }^{14}$, Bo Li ${ }^{15}$
}

1Department of Breast Surgery, Peking Union Medical College Hospital, Peking Union Medical College, Chinese Academy of Medical Sciences, Beijing, China

2Epidemiology and Statistics, Institute of Basic Medical Sciences Chinese Academy of Medical Sciences, School of Basic Medicine Peking Union Medical College, Beijing, China

3The Cancer Hospital, Tianjin Medical University, Tianjin, China

${ }^{4}$ YingBin Surgery Hospital of Yancheng, Jiangsu, China

${ }^{5}$ The General Hospital, Beijing Military Area Command, Beijing, China

"Southwest Hospital, the Third Military Medical University, Chongqing, China

${ }^{7}$ The Cancer Hospital, Fudan University, Shanghai, China

${ }^{8}$ Beijing ChaoYang Hospital, the Capital Medical University, Beijing, China

${ }^{9}$ Population and Family Planning Service Center of Zhuhai, Guangdong, China

10ShanXi Traditional Medicine Hospital, Shanxi, China

${ }^{11}$ Chinese 307 Hospital the People's Liberation Army, Beijing, China

12The Cancer Institute and Hospital, Peking Union Medical College Hospital, Chinese Academy of Medical Sciences, Beijing, China

${ }^{13}$ Maternity and Child Care Center of Qinhuangdao, Hebei, China

${ }^{14}$ First Hospital, Peking University, Beijing, China

${ }^{15}$ Beijing Hospital, Ministry of Health, Beijing, China

Submitted: 2 September 2010

Accepted: 11 January 2011

Arch Med Sci 2012; 8, 2: 303-309

DOI: 10.5114/aoms.2012.28558

Copyright @ 2012 Termedia \& Banach

\section{Abstract}

Introduction: To screen the risk factors associated with breast cancer among Chinese women in order to evaluate the individual risk of developing breast cancer among women in China.

Material and methods: A case-control study on 416 breast cancer patients and 1156 matched controls was conducted in 14 hospitals in 8 provinces of China in 2008. Controls were age- and region-matched to the cases. Clinicians conducted in-person interviews with the subjects to collect information on demographics and suspected risk factors for breast cancer that are known worldwide. Conditional logistic regression was used to derive odds ratios (OR) and 95\% confidence intervals $(\mathrm{Cl})$ for the associations between risk factors and breast cancer.

Results: Compared with matched controls, women with breast cancer were significantly more likely to have higher body mass index (BMI, OR $=4.07,95 \% \mathrm{Cl}$ : $2.98-5.55)$, history of benign breast disease (BBD) biopsy $(\mathrm{OR}=1.68,95 \% \mathrm{Cl}$ : 1.19-2.38), older age of menarche (AOM) $(\mathrm{OR}=1.41,95 \% \mathrm{Cl}$ : 1.07-1.87), stress anticipation (SA), for grade $1-4, \mathrm{OR}=2.15,95 \% \mathrm{Cl}: 1.26-3.66$; for grade $5-9, \mathrm{OR}=3.48$, $95 \% \mathrm{Cl}: 2.03-5.95)$ and menopause $(\mathrm{OR}=2.22,95 \% \mathrm{Cl}: 1.50-3.282)$ at the level of $p<0.05$. Family history of breast cancer (FHBC) in first-degree relatives $(\mathrm{OR}=1.66$, $95 \% \mathrm{Cl}: 0.77-3.59)$ and use of oral contraceptives (OC) (OR $=1.59,95 \% \mathrm{Cl}: 0.83-3.05)$ were associated with an increased risk of breast cancer at the level of $p<0.20$. Conclusions: Our results showed that BMI, history of BBD biopsy, older AOM, SA and menopause were associated with increased risk of breast cancer among Chinese women. The findings derived from the study provided some suggestions for population-based prevention and control of breast cancer in China.

Key words: breast cancer, risk factors, case-control study.

\author{
Corresponding author: \\ Prof. Qiang Sun MD, PhD \\ Peking Union Medical \\ College Hospital \\ No. 1 ShuaiFuYuan HoTong \\ 100730 Beijing, China \\ Phone: +86-10-88068140 \\ E-mail: \\ pumchsunqiang@126.com
}


Y.L. Xu, Q. Sun, G.L. Shan, J. Zhang, H.B. Liao, S.Y. Li, J. Jiang, Z.M. Shao, H.C. Jiang, N.C. Shen, Y. Shi, C.Z. Yu, B.N. Zhang, Y.H. Chen, X.N. Duan, B. Li

\section{Introduction}

In the past few decades, the incidence of breast cancer has increased worldwide, and major advances have been made in screening for risk factors associated with breast cancer in high-prevalence areas. Knowing the risk factors, healthcare providers are able to supply women with comparatively accurate information regarding their individual risk of developing breast cancer in western countries [1], where cancer risk assessment has emerged as an important component of cancer risk counseling [2-4]. The Gail model, which is based on breast cancer risk factors, has been shown to be well calibrated with respect to predicting the number of cancers likely to develop within cohorts of white American women with specific risk factors [5-7].

Studies focused on breast cancer risk factors have screened many well-known risk factors associated with breast cancer based on large sample sizes in western countries, and the magnitude of the associations have varied according to risk factors $[8,9]$. However, these risk factors are not based on Chinese women, and they cannot be applied in China directly because different populations may have different risk factors [10-12]. In China, much attention has been paid to breast cancer risk factors, and several case-control studies have been conducted to screen potential risk factors in various local areas, but most have used small sample sizes. Hence the breast cancer risk factors screened by foreign and domestic studies have not been comprehensive in reflecting the risk factors associated with Chinese women nationwide, and the Chinese government and clinicians cannot use those studies to identify populations at high risk for breast cancer.

Given the paucity of information regarding risk factors associated with breast cancer based on comparatively large sample sizes that are representative of the Chinese population, we investigated the risk factors through the project of breast cancer prediction (BCPC) in China. The risk factors established in our study will allow for the identification of Chinese women who are at increased risk of breast cancer. Also, effective early detection and disease prevention interventions may be implemented, and a model similar to the Gail model will be set up for large-scale screening in China.

\section{Material and methods}

The project of BCPC in China was one of the goals of China's eleventh 5-year plan, and it was approved by the local ethical committee. The project was designed to screen risk factors associated with breast cancer among Chinese women and to predict the individual risk of suffering from breast cancer through the established risk factors. The project was started in January and closed in July
2008. It was a multi-center, population-based, casecontrol study of incident breast cancer. Fourteen hospitals located in 8 provinces of China, where there were no nationally recognized screening services for breast cancer, participated in the study. Briefly, cases were aged 30 to 65 years, and they were identified upon receiving surgery at one of the 14 hospitals; the diagnoses of breast cancer (invasive or in situ) were confirmed through pathological tests. Cases were ineligible for the study if they were not permanent residents of the city where the hospitals were located or if they had a prior history of cancer. Three controls were randomly selected from healthy female permanent residents of each study area and were frequency-matched to each case based on similar demographics and behaviors during at least 5 years and during age intervals of 5 years. Females with a prior history of breast cancer or with a breast biopsy of unknown outcome were excluded from the study. Neighbors and friends were usually selected as the controls for each case. A total of 416 cases and 1156 controls were recruited between January and July 2008. Women older than 65 years were excluded because of the increased likelihood of co-morbidities, which affected the accuracy of the questionnaire responses.

Written informed consent was routinely obtained from all participants by doctors as part of the interview, and all relevant information was analyzed without patient identifiers in this study. Through a comparatively complete search in PubMed, a questionnaire that incorporated as many as possible known risk factors associated with breast cancer was developed. The items included in the questionnaire were as follows: demographic characteristics [13-18]; weight [13]; body mass index (BMI) (calculated using current weight in kilograms divided by standing height in meters squared as measured by a trained nurse) [7, 19]; reproductive history $[13,16,20]$; history of benign breast disease (BBD) biopsy and chronic diseases [13-16, 20]; FHBC $[13,14,16,20]$; menstrual status (including $A O M$, dysmenorrheal and menopause or not) $[13,14,16$, 20]; lifestyle behaviors, including reduced physical activity $[7,19]$, diets rich in fat/fresh meat/vitamins/garlic/onion [21-23], alcohol intake [22], smoking [24, 25], stress anticipation (SA) [26], education status [27], exposure to X-rays [28], history of breast feeding [29], use of oral contraceptives (OC) [30,31] or hormone replacement therapy (HRT) [14]; and mammogram breast density (BD) [14, 20, 32-35]. The above information was collected through inperson interviews. All of the participants were interviewed in a quiet environment with plenty of time to help the patients accurately recollect the information. The option of 'Uncertainty' was designed for each questionnaire item in case any of the participants was unable to recall the truth. 


\section{Statistical analysis}

Statistical tests were based on two-sided probabilities with a significant $\alpha<0.05$. Baseline differences in the characteristics between cases and controls were assessed using a $\chi^{2}$ test for categorical variables and a $t$-test for continuous variables. Conditional logistic regression was used to estimate the odds ratios (OR) and $95 \% \mathrm{Cl}$ to measure the association between the risk factors and risk of breast cancer. Subjects with missing values accounted for less than $0.5 \%$ of the total and were excluded from all analyses. All data analyses were performed using SAS 9.1 (SAS Institute Inc., Cary, NC, USA).

\section{Results}

The characteristics of cases and controls in the study are presented in Table I. The average age was $46.89 \pm 8.66$ years for cases and $46.17 \pm 8.56$ years for controls $(p=0.14)$. Compared to controls, cases were more likely to have higher BMI, stress anticipation, history of BBD biopsy, older AOM, menopause, HRT and lower intake of onion and garlic. First-degree FHBC and use of OC, which have been shown to be risk factors for breast cancer in other studies $[36,37]$, were not significantly associated with breast cancer in our study at the $p<0.05$ level.

Considering the mutual effects of different risk factors, we used conditional multiple logistic regression to analyze the breast cancer risk factors in China. The risk factors, including BMI, history of BBD biopsy, first-degree FHBC, breastfeeding, menstrual cycles (days), AOM, age at first live birth, SA, history of OC, HRT and menopause, were included in the conditional multiple logistic regression according to clinical experience and previous studies. The BMI ( $\geq 24)$, history of BBD biopsy, AOM ( $\geq 14$ years), $S A$ and menopause were significantly associated with increased risk of breast cancer at the level of $p<0.05$. After deleting the insignificant risk factors, conditional multiple logistic regression analysis was used again for the significant risk factors, and the results are shown in detail in Table II and Figure 1.

\section{Discussion}

To our knowledge, this is the first large-scale study investigating breast cancer risk factors among Chinese women, a population with low incidence of breast cancer. The eight provinces were scattered throughout China and differed greatly in economic status. For each hospital, information for at least 30 cases and corresponding controls was collected. Therefore, our study successfully screened breast cancer risk factors among Chinese women, and the results identified representative breast cancer risk factors in China. Consistent with previous case-control studies in other populations, we found an increase in the risk of breast cancer among Chinese women reporting higher BMI, history of BBD biopsy, later AOM, SA and menopause. The risk factors reported in other studies, such as higher education and occupational levels, no or few live births and short breastfeeding duration, were not found to be significant in our study, which might be reflective of the different populations examined.

The status of SA might be associated with many diseases including breast cancer, and much attention had been paid to the condition. Oerlemans et al. [38] tested the hypothesis that depression is a possible factor influencing the course of cancer by reviewing prospective epidemiological studies in the Netherlands and suggested a tendency towards a small and marginally significant association between depression and subsequent overall cancer risk and towards a stronger increase of breast cancer risk emerging many years after prior depression. Wakai et al. [39] prospectively examined the association between psychological factors and the risk of breast cancer in a non-Western population (Japanese) and suggested that having "ikigai" (Japanese term meaning something that makes one's life worth living) and being decisive were associated with a decrease in an individual's subsequent risk of breast cancer. In our study, the status of SA was evaluated by the interviewee themselves using a scale of grade 0-9, where 0 represented no depression and 9 represented the most depressed state. The newly diagnosed cases were evaluated according to their mood at 3 months before the diagnosis of breast cancer, and the controls were evaluated according to their mood at 3 months before the interview with the clinicians. Compared with the group of grade 0 , the groups of grade 1-4 and grade 5-9 presented a significant association with the risk of breast cancer, while the group of grade 5-9 had a stronger increase in breast cancer risk. In addition, our study determined the overall stress anticipation status by self-evaluation, which is different from previous studies.

The $A O M \geq 14$ was associated with increased risk of breast cancer, and this result seemed to contradict previous studies. Several studies $[13,14,18,20]$ outside China have concluded that younger AOM raised the risk of breast cancer. However, Ozmen et al. [40] conducted a nested case-control study in Turkey to clarify the risk factors for breast cancer in developing countries, and AOM was not identified as a risk factor for breast cancer. Based on a case-control study in the city of Taipei, Wu et al. [41] suggested that together with elevated IGF-I concentrations, a prolonged interval of estrogen exposure was associated with significantly increased risk of breast cancer, particularly among 
Y.L. Xu, Q. Sun, G.L. Shan, J. Zhang, H.B. Liao, S.Y. Li, J. Jiang, Z.M. Shao, H.C. Jiang, N.C. Shen, Y. Shi, C.Z. Yu, B.N. Zhang, Y.H. Chen, X.N. Duan, B. Li

Table I. Comparison of demographic and breast cancer risk factors between cases and controls, BCPC $(n=1572)$

\begin{tabular}{|c|c|c|c|}
\hline Characteristics & $\begin{array}{c}\text { Cases }(n=416) \\
\%\end{array}$ & $\begin{array}{c}\text { Controls }(n=1156) \\
\%\end{array}$ & Value of $p^{a}$ \\
\hline Han race & 97.58 & 96.39 & 0.25 \\
\hline \multicolumn{4}{|l|}{ Education } \\
\hline None/elementary/high school & 71.57 & 69.24 & \\
\hline Professional/college+ & 28.43 & 30.76 & 0.38 \\
\hline \multicolumn{4}{|l|}{ Occupation status } \\
\hline Physical work & 55.42 & 50.18 & \\
\hline Mental work & 44.58 & 49.82 & 0.07 \\
\hline \multicolumn{4}{|l|}{$\mathrm{BMI}\left[\mathrm{kg} / \mathrm{m}^{2}\right]$} \\
\hline Mean \pm SD & $23.77 \pm 3.60$ & $23.21 \pm 2.93$ & 0.01 \\
\hline$\geq 24$ & 41.71 & 34.93 & 0.05 \\
\hline \multicolumn{4}{|l|}{ Stress anticipation } \\
\hline Grade $0^{b}$ & 4.23 & 7.4 & \\
\hline Grade 1-4 & 46.27 & 51.9 & 0.11 \\
\hline Grade 5-9 & 49.5 & 40.7 & 0.01 \\
\hline First-degree FHBC & 8.66 & 6.24 & 0.1 \\
\hline Ever had any biopsy of BBD & 14.57 & 6.46 & $<0.001$ \\
\hline $\mathrm{AOM}($ mean $\pm \mathrm{SD})$ & $14.33 \pm 1.809$ & $14.10 \pm 1.704$ & 0.02 \\
\hline $\mathrm{AOM}(\geq 14)$ & 64.32 & 58.33 & 0.03 \\
\hline Menstrual cycle, days (mean \pm SD) & $28.62 \pm 3.98$ & $28.64 \pm 4.23$ & 0.92 \\
\hline Use of OC & 5.56 & 3.91 & 0.16 \\
\hline Ever had a live birth & 95.86 & 94.16 & 0.19 \\
\hline Number of live births (mean $\pm \mathrm{SD}$ ) & $1.36 \pm 0.67$ & $1.30 \pm 0.68$ & 0.12 \\
\hline Age at first live birth (mean \pm SD) [years] & $25.52 \pm 4.03$ & $25.84 \pm 3.64$ & 0.17 \\
\hline Ever breastfed & 86.02 & 87.82 & 0.37 \\
\hline Months of breastfeeding (mean $\pm \mathrm{SD}$ ) & $14.39 \pm 10.44$ & $13.21 \pm 10.66$ & 0.09 \\
\hline Abortion & 56.11 & 55.88 & 0.94 \\
\hline Menopause & 43.33 & 33.43 & $<0.001$ \\
\hline Age at menopause (mean \pm SD) & $48.89 \pm 3.218$ & $49.33 \pm 3.169$ & 0.16 \\
\hline HRT & 5.43 & 2.45 & 0.003 \\
\hline Smoking & 2.16 & 2.78 & 0.50 \\
\hline Alcohol intake & 8.82 & 8.51 & 0.85 \\
\hline Exercise participation & 29.13 & 32.35 & 0.23 \\
\hline Dietary habits: vegetables & 93.01 & 95.37 & 0.07 \\
\hline Dietary habits: meat & 71.01 & 72.69 & 0.52 \\
\hline Dietary habits: onion and garlic & 56.04 & 64.16 & 0.004 \\
\hline
\end{tabular}

aDerived from $t$-tests for continuous variables and 2 test for categorical variables, ${ }^{b}$ grade 0 was the control group

estrogen-positive cases. The critical period of estrogen exposure was estimated by the interval between the AOM and age at first full-term pregnancy. Hence, it might be more reasonable to evaluate the association between breast cancer risk and the critical period of estrogen exposure (not AOM). However, we found no significant association between breast cancer risk and the critical period of estrogen exposure in our case-control study (data not shown). Although it is not definite whether AOM is a risk factor for Asians, other factors might interact with AOM; thus, other studies are needed to further clarify these results. The results of our study could be referred to by future relevant studies. 
The well-known risk factor of FHBC among firstdegree relatives was not significantly associated with the risk of breast cancer in our study at the level of $\alpha=0.05$. At the level of $\alpha=0.20$, family history of breast cancer (FHBC) among first-degree relatives was significantly associated with the risk of breast cancer. Kilfoy et al. [36], based on a population-based cohort study in Shanghai, China, suggested that the risk of breast cancer was elevated $(\mathrm{RR}=1.74,95 \% \mathrm{Cl}: 1.10-2.73)$ for those with a FHBC in first-degree relatives, and the risk was stronger for women who were younger than 55 years $(R R=$ 2.07, 95\% Cl: 1.17-3.64). The cohort study also suggested that the link between breast cancer risk and family history of lung and esophageal cancers as well as leukemia warranted further investigation. In our study, family cancer history in first-/second/third-degree relatives was investigated in cases and controls. No link was observed between breast cancer risk and family history of cancers (except breast cancer) in first-/second-/third- degree relatives (data not shown). For FHBC, no link was observed between the risk of breast cancer and family history of breast cancer in second-/third-degree relatives (data not shown), and FHBC in first-degree relatives was associated with the risk of breast cancer at the level of $\alpha=0.20$. An explanation for the different results between these two studies might be the socio-economic differences between the populations. Our study was conducted in eight provinces throughout China including people of greatly different economic status, while the cohort study conducted by Kilfoy et al. was limited to the area of Shanghai, where socio-economic status is high in China. High socio-economic status might affect the magnitude of FHBC. A study conducted by Verkooijen et al. [42] in Switzerland confirmed that a positive $\mathrm{FHBC}$ may reduce socio-economic status differences in the access to screening and optimal treatment. Socio-economic status differences objectively existed in China and may explain the significant association that was established at the $\alpha$ level of 0.20 rather than 0.05 .

The association between the use of $O C$ and the risk of breast cancer has been controversial. Based on the $\chi^{2}$ test and multivariate logistic regression analysis, Ozmen et al. [40] suggested that history of OC significantly decreased the risk of breast cancer. Rosenberg et al. [37] suggested that OC use ( $\geq 12$ months) is associated with an increased risk of breast cancer diagnosed in recent years, and the association of OC use with breast cancer risk did not differ according to estrogen or progesterone receptor status of the tumor. Hence, the duration of $\mathrm{OC}$ use should be considered when analyzing the risk factor. We investigated the association between OC use ( $\geq 12$ months) and the risk of breast cancer, and we found a significant association at the $\alpha$ lev-
Table II. Multiple logistic regression of breast cancer risk factors in China

\begin{tabular}{|c|c|c|c|c|}
\hline Risk factors & Value of $\chi^{2}$ & Value of $p$ & OR & $95 \% \mathrm{Cl}$ \\
\hline BMIa & 77.66 & $<0.0001$ & 4.07 & $2.98-5.55$ \\
\hline BBD biopsy & 8.62 & 0.003 & 1.68 & $1.19-2.38$ \\
\hline $\mathrm{AOM}^{\mathrm{b}}$ & 5.94 & 0.02 & 1.41 & $1.07-1.87$ \\
\hline SA $1^{c}$ & 7.87 & 0.005 & 2.15 & $1.26-3.66$ \\
\hline $\mathrm{SA} 2$ & 20.67 & $<0.0001$ & 3.48 & $2.03-5.95$ \\
\hline Menopause & 15.93 & $<0.0001$ & 2.22 & $1.50-3.28$ \\
\hline
\end{tabular}

a24 as the cut point, b14 years as the cut point, CSA1 represented grade $1-4$, and 2 represented grade 5-9. Grade 0 was used as the control

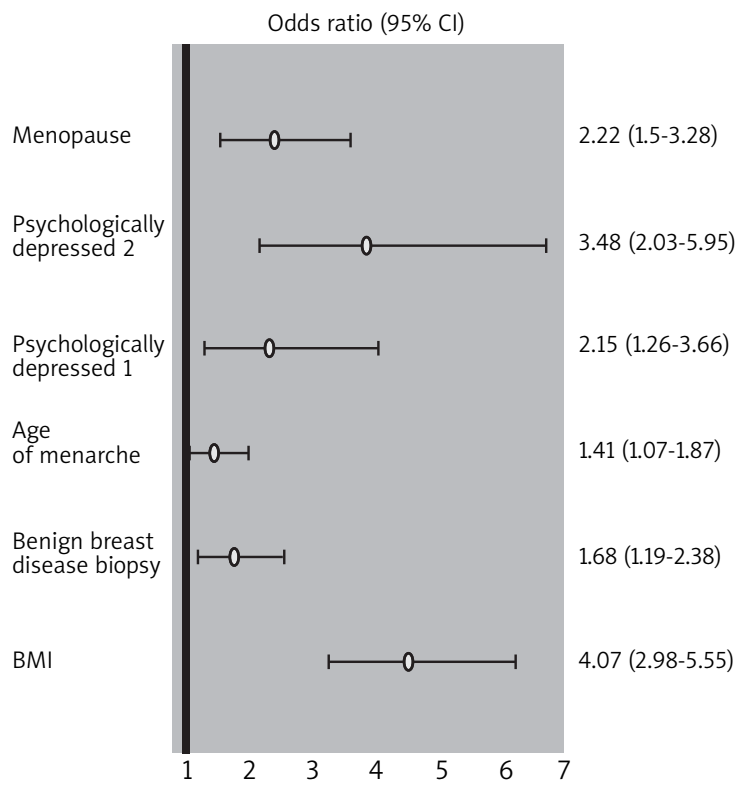

Figure 1. Forest plot of results from multiple logistic regression of breast cancer risk factors in China

el of 0.20 rather than 0.05 . Folger et al. [43] estimated the association between breast cancer risk and short-term ( $<6$ months) OC use and also explored the variation in the risk estimates by using patient characteristics and medical, menstrual and reproductive histories. This study indicated that short-term OC use was not associated with breast cancer risk (OR $=1.00,95 \% \mathrm{Cl}$ : 0.80-1.10), while the underlying characteristics of users or other unmeasured factors influenced the duration of use and breast cancer risk. Therefore, the association between $\mathrm{OC}$ use and breast cancer risk was affected by the duration of OC use and other underlying factors.

As to the risk factor of $B D$, no definite result was obtained. There are 3 reasons for this: firstly, the mammogram in China was not as popular as in developed countries, so the majority of interviewees have never received a mammogram examination and have no information relevant to $\mathrm{BD}$; sec- 
ondly, the diagnostic criteria of the mammogram have not been identical all over China, and more training is needed for roentgenologists; thirdly, because of the above 2 reasons, little reliable information associated with BD has been collected by questionnaires and it cannot be used to make an analysis. As to the factor of dietary habits, published literatures [21-23] have indicated that dietary habits have a correlation with risk of breast cancer. As Chinese people do not have settled dietary habits, it is difficult to establish the daily intake of vegetables, meat, onion/garlic and so on. In order to collect information on dietary habits and intake frequency of food, we designed four options for interviewees to choose: A: never, B: once per month or less, C: three times per week or more, D: between $B$ and $C$. Finally we divided the data into 2 groups: never or ever. The results indicated that higher intake of onion and garlic might have a correlation with decreased breast cancer risk. However, as a retrospective study, together with comparatively little attention paid to dietary habits by Chinese women, the result warrants more research. In addition, diet rich in fresh meat should be further studied. It is a pity that the factors of $\mathrm{BD}$ and dietary habits have not been fully investigated in our study.

There are several models currently available for predicting the individual risk of breast cancer based on known risk factors, including the Gail model, the Gail-2 model, the Claus model, the Claus tables, BOADICEA, the Jonker model, the Claus-Extended formula and the Tyrer-Cuzick model [5-7, 44-50]. These models were derived from different studies and were thus adapted to their corresponding populations. The risk of breast cancer has a correlation with genic and extragenic factors [51, 52], and the Gail model identifies women with increased breast cancer risk due to non-familial risk factors and genetic influences. Our study clarified the extragenic risk factors of breast cancer among Chinese women, and the results may be promising for predicting breast cancer risk in Chinese women. A predictive model could be developed to evaluate the individual risk of breast cancer in Chinese women and to offer effective early detection and disease prevention interventions for women with increased risk of breast cancer.

\section{References}

1. Hoskins KF, Zwaagstra A, Ranz M. Validation of a tool for identifying women at high risk for hereditary breast cancer in population-based screening. Cancer 2006; 107: 1769-76.

2. Armstrong K, Eisen A, Weber B. Assessing the risk of breast cancer. N Engl J Med 2000; 342: 564-71.

3. Domchek SM, Eisen A, Calzone K, et al. Application of breast cancer risk prediction models in clinical practice. J Clin Oncol 2003; 21: 593-601.

4. Freedman AN, Seminara D, Gail MH, et al. Cancer risk prediction models: a workshop on development, evaluation, and application. J Natl Cancer Inst 2005; 97 : 715-23.

5. van Asperen CJ, Jonker MA, Jacobi CE, et al. Risk estimation for healthy women from breast cancer families: new insights and new strategies. Cancer Epidemiol Biomarkers Prev 2004; 13: 87-93.

6. Jacobi CE, de Bock GH, Siegerink B, et al. Differences and similarities in breast cancer risk assessment models in clinical practice: which model to choose? Breast Cancer Res Treat 2009; 115: 381-90.

7. Euhus DM. Understanding mathematical models for breast cancer risk assessment and counseling. Breast J 2001; 7: 224-32.

8. Petram C, Ames RC, Sandhya P. Oral contraceptive use and the risk of breast cancer. Mayo Clin Proc 2008; 83: 86-91.

9. Carol S, Kathy BB, Tim B, et al. Reproductive history in relation to breast cancer risk among Hispanic and nonHispanic white women. Cancer Causes Control 2008; 19: 391-401.

10. Tjia J, Micco E, Armstrong K. Interest in breast cancer chemoprevention among older women. Breast Cancer Res Treat 2008; 108: 435-53.

11. Euhus DM, Bu D, Ashfaq R, et al. Atypia and DNA methylation in nipple duct lavage in relation to predicted breast cancer risk. Cancer Epidemiol Biomarkers Prev 2007; 16: 1812-21.

12. Cazzaniga M, Severi G, Casadio C, et al. Atypia and Ki-67 expression from ductal lavage in women at different risk for breast cancer. Cancer Epidemiol Biomarkers Prev 2006; 15: 1311-5.

13. Brinton LA, Sherman ME, Carreon JD, et al. Recent trends in breast cancer among younger women in the United States. J Natl Cancer Inst 2008; 100: 1643-8.

14. Vachon CM, van Gils CH, Sellers TA, et al. Mammographic density, breast cancer risk and risk prediction. Breast Cancer Res 2007; 9: 217-25.

15. Boyd NF, Guo H, Martin LJ, et al. Mammographic density and the risk and detection of breast cancer. N Engl J Med 2007; 356: 227-36.

16. Barlow WE, White E, Ballard-Barbash R, et al. Prospective breast cancer risk prediction model for women undergoing screening mammography. J Natl Cancer Inst 2006; 98: 1204-14.

17. Rockhill B, Spiegelman D, Byrne C, et al. Validation of the Gail et al. model of breast cancer risk prediction and implications for chemoprevention. J Natl Cancer Inst 2001; 93: 358-66.

18. Palomares MR, Machia JRB, Lehman CD, et al. Mammographic density correlation with Gail model breast cancer risk estimates and component risk factors. Cancer Epidemiol Biomarkers Prev 2006; 15: 1324-30.

19. Anderson WF, Matsuno RK, Sherman ME, et al. Estimating age-specific breast cancer risks: a descriptive tool to identify age interactions. Cancer Causes Control 2007; 18: 439-47.

20. Santen RJ, Boyd NF, Chlebowski RT, et al. Critical assessment of new risk factors for breast cancer: considerations for development of an improved risk prediction model. Endocr Rel Cancer 2007; 14: 169-87.

21. Chen J, Pee D, Ayyagari R, et al. Projecting absolute invasive breast cancer risk in white women with a model that includes mammographic density. J Natl Cancer Inst 2006; 98: 1215-26.

22. Kelemen LE, Pankratz VS, Sellers TA, et al. Age-specific trends in mammographic density The Minnesota Breast Cancer Family Study. Am J Epidemiol 2008; 167: 1027-36. 
23. Huo D, Adebamowo CA, Ogundiran TO, et al. Parity and breastfeeding are protective against breast cancer in Nigerian women. Br J Cancer 2008; 98: 992-6.

24. Suzuki S, Kojima M, Tokudome S, et al. Effect of physical activity on breast cancer risk: findings of the Japan Collaborative Cohort Study. Cancer Epidemiol Biomarkers Prev 2008; 17: 339-401.

25. Pirie K, Beral V, Peto R, et al. Passive smoking and breast cancer in never smokers: prospective study and metaanalysis. Int J Epidemiol 2008; 37: 1069-79.

26. Cui XH, Dai Q, Tseng M, et al. Dietary patterns and breast cancer risk in the Shanghai Breast Cancer Study. Cancer Epidemiol Biomarkers Prev 2007; 16: 1443-8.

27. Mahoney MC, Bevers T, Linos E, et al. Opportunities and strategies for breast cancer prevention through risk reduction. CA Cancer J Clin 2008; 58: 347-71.

28. Wellisch DK, Lindberg NM. A psychological profile of depressed and nondepressed women at high risk for breast cancer. Psychosomatics 2001; 42: 330-6.

29. Mouw T, Koster A, Wright ME, et al. Education and risk of cancer in a large cohort of men and women in the United States. Available at: www.plosone.org 2008; 3: e3639.

30. Bremnes Y, Ursin G, Bjurstam N, et al. Different measures of smoking exposure and mammographic density in postmenopausal Norwegian women: a cross-sectional study. Breast Cancer Res 2007; 9: 1/8.

31. Cardis E, Hall J, Tavtigian SV. Identification of women with an increased risk of developing radiation-induced breast cancer. Breast Cancer Res 2007; 9: 106.

32. Sant M, Francisci S, Capocaccia R, et al. Time trends of breast cancer survival in Europe in relation to incidence and mortality. Int J Cancer 2006; 119: 2417-22.

33. Karliner LS, Napoles-Springer A, Kerlikowske K, et al. Missed opportunities: family history and behavioral risk factors in breast cancer risk assessment among a multiethnic group of women. J Gen Intern Med 2007; 22: 308-14.

34. Gail MH, Costantino JP. Validating and improving models for projecting the absolute risk of breast cancer. J Natl Cancer Inst 2001; 93: 334-5.

35. Gail MH, Benichou J. Epidemiology and biostatistics program of the National Cancer Institute. J Natl Cancer Inst 1994; 86: 573-5.

36. Kilfoy BA, Zhang Y, Shu XO, et al. Family history of malignancies and risk of breast cancer: prospective data from the Shanghai women's health study. Cancer Causes Control 2008; 19: 1139-45.

37. Rosenberg L, Zhang Y, Coogan PF, et al. A case-control study of oral contraceptive use and incident breast cancer. Am J Epidemiol 2009; 169: 473-9.

38. Oerlemans ME, van den Akker M, Schuurman AG, et al. A meta-analysis on depression and subsequent cancer risk. Clin Pract Epidemol Ment Health 2007; 3: 29.

39. Wakai K, Kojima M, Nishio K, et al. Psychological attitudes and risk of breast cancer in Japan: a prospective study. Cancer Causes Control 2007; 18: 259-67.

40. Ozmen V, Ozcinar B, Karanlik H, et al. Breast cancer risk factors in Turkish women - a University Hospital based nested case control study. World J Surg Oncol 2009; 7: 37.

41. Wu MH, Chou YC, Chou WY, et al. Relationships between critical period of estrogen exposure and circulating levels of insulin-like growth factor-I (IGF-I) in breast cancer: evidence from a case-control study. Int J Cancer 2010; 126: 508-14.

42. Verkooijen HM, Rapiti E, Fioretta G, et al. Impact of a positive family history on diagnosis, management, and survival of breast cancer: different effects across socio-economic groups. Cancer Causes Control 2009; 20: 1689-96.
43. Folger SG, Marchbanks PA, McDonald JA, et al. Risk of breast cancer associated with short-term use of oral contraceptives. Cancer Causes Control 2007; 18: 189-98.

44. Bondy ML, Newman LA. Assessing breast cancer risk: evolution of the gail model. J Natl Cancer Inst 2006; 98 : 1172-3.

45. Adams-Campbell LL, Makambi KH, Palmer JR, et al. Diagnostic accuracy of the gail model in the Black Women's Health Study. Breast J 2007; 13: 332-6.

46. Decarli A, Calza S, Masala G, et al. Gail model for prediction of absolute risk of invasive breast cancer: independent evaluation in the Florence - European Prospective Investigation Into Cancer and Nutrition Cohort. J Natl Cancer Inst 2006; 98: 1686-93.

47. Claus EB, Risch N, Thompson WD. Genetic analysis of breast cancer in the cancer and steroid hormone study. Am J Hum Genet 1991; 48: 232-42.

48. Fisher B, Costantino JP, Wicherham DL, et al. Tamoxifen for prevention of breast cancer: Report of the National Surgical Adjuvant Breast and Bowel Project P-1 Study. J Natl Cancer Inst 1998; 90: 1371-88.

49. Jonker MA, Jacobi CE, Hoogendoorn WE, et al. Modeling familial clustered breast cancer using published data. Cancer Epidemiol Biomarkers Prev 2003; 12: 1479-85.

50. Antoniou AC, Pharoah PPD, Smith P, et al. The BOADICEA model of genetic susceptibility to breast and ovarian cancer. Br J Cancer 2004; 91: 1580-90.

51. Hosseini M, Houshmand M, Ebrahimi A. The ERCC2k751 polymorphism is associated with breast cancer risk. Arch Med Sci 2009; 5: 456-8.

52. Hosseini M, Houshmand M, Ebrahimi A. Breast cancer risk not only was not associated with CYP17/A2 allele but also was related to A1 allele. Arch Med Sci 2009; 5: 103-6. 